\title{
Correcting Rate Constants from Anharmonic Molecular Dynamics for Quantum Effects
}

\author{
Felix Schmalz, Wassja A. Kopp, Leif C. Kröger and Kai Leonhard* \\ Chair of Technical Thermodynamics, \\ RWTH Aachen University, Aachen, Germany
}

December 19, 2019

*To whom correspondence should be adressed: kai.leonhard@ltt.rwth-aachen.de; felix.schmalz@ltt.rwth-aachen.de 


\section{The Pitzer-Gwinn Approximation Applied to a Co- sine Potential}

Our cosine potential is given by

$$
V(x)=\frac{V_{0}}{2} \cos (x)+\frac{V_{0}}{2}
$$

For the test, we use a one-dimensional oscillator with a moment of inertia obtained from the torsional mode in butane (ethyl group vs. ethyl group). From that, we derived a minimal working example of a simple cosine potential as a special case of Fourier functions that are a reasonable representation of a $1 \mathrm{D}$ hindered rotation potential. The classical partition function is known exactly and the quantum-mechanical $(\mathrm{QM})$ partition function $Q_{\mathrm{q}}^{\text {anh }}$ can be computed with the program package TAMkin. The classical partition function $Q_{\mathrm{c}}^{\text {anh }}$ can be found by integration and is related to the modified Bessel function of the first kind $I_{0}$ :

$$
\begin{aligned}
Q_{\mathrm{c}}^{\mathrm{anh}} & =\sqrt{2 \pi I / \beta h^{2}} \int_{0}^{2 \pi} \exp \left(-\beta\left(V_{0} / 2+V_{0} / 2 \cos (\theta)\right)\right) \mathrm{d} \theta \\
& =\sqrt{2 \pi I / \beta h^{2}} 2 \pi I_{0}\left(-\beta V_{0} / 2\right) \exp \left(-\beta V_{0} / 2\right)
\end{aligned}
$$

With $Q_{\mathrm{c}}^{\mathrm{HO}}=(\beta h \nu)^{-1}$ and $Q_{\mathrm{q}}^{\mathrm{HO}}=(1-\exp (-\beta h \nu))^{-1}$ and $\nu$ as the harmonic frequency, the Pitzer-Gwinn (PG) relation can be evaluated. The numerical results can be found in Table S1. The results prove the very good applicability of the PG approximation, but show some underestimation of the exact partition function at low temperatures $\left(T \approx 100 \mathrm{~K}\right.$ and high barriers $\left.V_{0} \approx 200 \mathrm{~kJ} / \mathrm{mol}\right)$ while the error is still below $3 \%$. The approximation also works for extreme cases, where the harmonic frequency $\nu$ is very low. At high temperatures, the PG approximation works especially well. 


\begin{tabular}{rrlll}
$V_{0}$ & $\nu$ & \multicolumn{2}{c}{$\left(Q_{\mathrm{q}}^{\mathrm{HR}} / Q_{\mathrm{q}}^{\mathrm{HO}}\right) /\left(Q_{\mathrm{c}}^{\mathrm{HR}} / Q_{\mathrm{c}}^{\mathrm{HO}}\right)$} \\
\cline { 3 - 6 }$(\mathrm{kJ} / \mathrm{mol})$ & $\left(\mathrm{cm}^{-1}\right)$ & $100 \mathrm{~K}$ & $1000 \mathrm{~K}$ & $4000 \mathrm{~K}$ \\
\hline 0.01 & 1.1 & 0.9997 & 0.9996 & 0.9996 \\
0.1 & 3.6 & 0.9996 & 0.9996 & 0.9996 \\
1 & 11.4 & 0.9988 & 0.9996 & 0.9996 \\
4 & 22.8 & 0.9983 & 0.9996 & 0.9996 \\
16 & 45.5 & 0.9974 & 0.9994 & 0.9995 \\
64 & 91.0 & 0.9930 & 0.9990 & 0.9994 \\
256 & 182.1 & 0.9757 & 0.9973 & 0.9990 \\
\hline
\end{tabular}

Table S1: Accuracy of the Pitzer-Gwinn relation tested with a cosine potential for different barrier heigths $\left(V_{0}\right)$ and temperatures. HR and HO denote hindered rotor and harmonic oscillator respectively. The harmonic frequency $\nu$ that corresponds to a $V_{0}$ is also shown. The closer the value is to unity, the better the relation. A value of unity means that the relation is perfectly true. The relation holds up very good except for low temperatures and high barriers. 


\section{Statistical Basis of Rate Constants from MD}

\begin{tabular}{|c|c|c|c|c|c|}
\hline No & & $1600 \mathrm{~K}$ & $2600 \mathrm{~K}$ & $3000 \mathrm{~K}$ & $3400 \mathrm{~K}$ \\
\hline \multirow{2}{*}{1} & $\mathrm{f}$ & & 112 & 92 & 76 \\
\hline & b & & 3 & 22 & 23 \\
\hline \multirow{2}{*}{2} & $\mathrm{f}$ & & 49 & 77 & 87 \\
\hline & $\mathrm{b}$ & & 13 & 22 & 26 \\
\hline \multirow{2}{*}{3} & $\mathrm{f}$ & & 37 & 195 & 563 \\
\hline & b & & 111 & 313 & 757 \\
\hline \multirow{2}{*}{4} & $\mathrm{f}$ & & 0 & 18 & 49 \\
\hline & b & & 24 & 68 & 107 \\
\hline \multirow{2}{*}{5} & $\mathrm{f}$ & & 67 & 87 & 73 \\
\hline & b & & 7 & 6 & 7 \\
\hline Butanone $+\mathrm{HO} 2$ & & 2 & & & \\
\hline
\end{tabular}

Table S2: Reactions examined in this work and their number of forward (f) and backward (b) occurrences in the MD simulations during 20x10 ns of simulated time.

\section{Deviations from and between experiments}

Rate constants from various experimental sources are listed in columns $k_{\exp , i}$ of Table S3. The corresponding references can be found in the paper. Column "exp dev" contains the standard deviation of all available rate constants at a temperature and a specific reaction computed from Excel's stdev.p function evaluating $\sqrt{\frac{\sum\left(x-x_{\text {mean }}\right)^{2}}{n}}, n$ being the sample size. The function was applied to the logarithms of the rate constants. Similarly, column "corr dev" evaluates $10 \sqrt{\frac{\sum\left(k_{\mathrm{corr}}-k_{\text {exp }, \mathrm{ln}, \text { mean }}\right)^{2}}{n}}$ where $k_{\text {exp, ln, mean }}$ is 10 to the power of the mean value of the logarithm of the experimental rate constants. This yields a deviation factor of corrected rate constants to the mean value of experimental rate constants. The second-to-last row presents mean values of the corresponding columns, while the last row presents mean values where reactions 1 and 2 have been neglected. This is given because for these two reactions, the scheme proposed in the paper might not yield accurate results for reasons discussed in the paper. 


\begin{tabular}{|c|c|c|c|c|c|c|c|}
\hline $\mathrm{R}$ & $T / 1 \mathrm{~K}$ & $k_{\exp }$ & $k_{\exp , 2}$ & $k_{\exp , 3}$ & exp dev & $k_{\text {corr }}$ & corr dev \\
\hline \multirow[t]{3}{*}{$1 \mathrm{f}$} & 2600 & $1.722 \mathrm{E}+13$ & $3.155 \mathrm{E}+13$ & $3.886 \mathrm{E}+13$ & 1.412 & $1.662 \mathrm{E}+12$ & 0.060 \\
\hline & 3000 & $2.880 \mathrm{E}+13$ & $5.890 \mathrm{E}+13$ & $5.737 \mathrm{E}+13$ & 1.393 & $2.362 \mathrm{E}+12$ & 0.051 \\
\hline & 3400 & $4.405 \mathrm{E}+13$ & $9.793 \mathrm{E}+13$ & $7.730 \mathrm{E}+13$ & 1.398 & $3.889 \mathrm{E}+12$ & 0.056 \\
\hline \multirow[t]{3}{*}{ b } & 2600 & $6.473 \mathrm{E}+11$ & $1.251 \mathrm{E}+12$ & $2.425 \mathrm{E}+12$ & 1.715 & $6.013 \mathrm{E}+11$ & 0.480 \\
\hline & 3000 & $9.446 \mathrm{E}+11$ & $1.810 \mathrm{E}+12$ & $4.743 \mathrm{E}+12$ & 1.940 & $1.699 \mathrm{E}+12$ & 0.846 \\
\hline & 3400 & $1.261 \mathrm{E}+12$ & $2.401 \mathrm{E}+12$ & $8.323 \mathrm{E}+12$ & 2.189 & $1.757 \mathrm{E}+12$ & 0.599 \\
\hline \multirow[t]{3}{*}{$2 \mathrm{f}$} & 2600 & $3.238 \mathrm{E}+12$ & $9.925 \mathrm{E}+12$ & & 1.751 & $5.969 \mathrm{E}+12$ & 1.053 \\
\hline & 3000 & $4.781 \mathrm{E}+12$ & $1.598 \mathrm{E}+13$ & & 1.828 & $8.073 \mathrm{E}+12$ & 0.924 \\
\hline & 3400 & $6.441 \mathrm{E}+12$ & $2.300 \mathrm{E}+13$ & & 1.889 & $1.108 \mathrm{E}+13$ & 0.910 \\
\hline \multirow[t]{3}{*}{ b } & 2600 & $7.023 \mathrm{E}+12$ & $7.597 \mathrm{E}+12$ & & 1.040 & $3.581 \mathrm{E}+12$ & 0.490 \\
\hline & 3000 & $1.001 \mathrm{E}+13$ & $1.276 \mathrm{E}+13$ & & 1.129 & $4.571 \mathrm{E}+12$ & 0.404 \\
\hline & 3400 & $1.313 \mathrm{E}+13$ & $1.968 \mathrm{E}+13$ & & 1.224 & $4.720 \mathrm{E}+12$ & 0.294 \\
\hline \multirow[t]{3}{*}{$3 \mathrm{f}$} & 2600 & $3.236 \mathrm{E}+12$ & $3.773 \mathrm{E}+12$ & $4.382 \mathrm{E}+12$ & 1.132 & $1.173 \mathrm{E}+12$ & 0.311 \\
\hline & 3000 & $5.853 \mathrm{E}+12$ & $6.564 \mathrm{E}+12$ & $7.742 \mathrm{E}+12$ & 1.122 & $2.287 \mathrm{E}+12$ & 0.343 \\
\hline & 3400 & $9.210 \mathrm{E}+12$ & $1.003 \mathrm{E}+13$ & $1.197 \mathrm{E}+13$ & 1.115 & $3.961 \mathrm{E}+12$ & 0.383 \\
\hline \multirow[t]{3}{*}{ b } & 2600 & $8.164 \mathrm{E}+12$ & $1.477 \mathrm{E}+13$ & $1.596 \mathrm{E}+13$ & 1.349 & $8.279 \mathrm{E}+12$ & 0.665 \\
\hline & 3000 & $9.323 \mathrm{E}+12$ & $1.747 \mathrm{E}+13$ & $2.165 \mathrm{E}+13$ & 1.430 & $1.310 \mathrm{E}+13$ & 0.861 \\
\hline & 3400 & $1.032 \mathrm{E}+13$ & $1.986 \mathrm{E}+13$ & $2.798 \mathrm{E}+13$ & 1.513 & $1.921 \mathrm{E}+13$ & 1.073 \\
\hline \multirow[t]{3}{*}{$4 \mathrm{f}$} & 2600 & $8.403 \mathrm{E}+12$ & $1.871 \mathrm{E}+12$ & & 2.120 & & \\
\hline & 3000 & $1.386 \mathrm{E}+13$ & $2.173 \mathrm{E}+12$ & & 2.526 & $2.371 \mathrm{E}+13$ & 4.320 \\
\hline & 3400 & $2.125 \mathrm{E}+13$ & $2.436 \mathrm{E}+12$ & & 2.953 & $2.370 \mathrm{E}+13$ & 3.295 \\
\hline \multirow[t]{3}{*}{ b } & 2600 & $1.257 \mathrm{E}+13$ & $2.000 \mathrm{E}+13$ & $2.144 \mathrm{E}+13$ & 1.268 & $7.296 \mathrm{E}+12$ & 0.416 \\
\hline & 3000 & $2.186 \mathrm{E}+13$ & $3.364 \mathrm{E}+13$ & $3.580 \mathrm{E}+13$ & 1.245 & $2.367 \mathrm{E}+13$ & 0.796 \\
\hline & 3400 & $3.488 \mathrm{E}+13$ & $5.179 \mathrm{E}+13$ & $5.477 \mathrm{E}+13$ & 1.223 & $2.919 \mathrm{E}+13$ & 0.631 \\
\hline \multirow[t]{3}{*}{$5 \mathrm{f}$} & 2600 & $4.347 \mathrm{E}+13$ & $1.009 \mathrm{E}+14$ & $1.038 \mathrm{E}+14$ & 1.498 & $7.355 \mathrm{E}+13$ & 0.956 \\
\hline & 3000 & $5.699 \mathrm{E}+13$ & $1.296 \mathrm{E}+14$ & $1.462 \mathrm{E}+14$ & 1.520 & $5.889 \mathrm{E}+13$ & 0.574 \\
\hline & 3400 & $7.009 \mathrm{E}+13$ & $1.570 \mathrm{E}+14$ & $1.958 \mathrm{E}+14$ & 1.555 & $6.302 \mathrm{E}+13$ & 0.488 \\
\hline \multirow[t]{3}{*}{ b } & 2600 & $3.868 \mathrm{E}+11$ & & & & $3.920 \mathrm{E}+11$ & 1.013 \\
\hline & 3000 & $8.159 \mathrm{E}+11$ & & & & $3.912 \mathrm{E}+11$ & 0.480 \\
\hline & 3400 & $1.490 \mathrm{E}+12$ & & & & $4.153 \mathrm{E}+11$ & 0.279 \\
\hline Avg & & & & & 1.517 & & 2.649 \\
\hline \multicolumn{2}{|c|}{ Avg w/o R1, 2} & & & & 1.502 & & 2.084 \\
\hline
\end{tabular}

Table S3: Experimental benchmark rate constants and corrected rate constants obtained in this study. Units of rate constants are $\mathrm{cm}^{3}$, mol and s. 
Harmonic Frequencies of Species and Transition States 


\begin{tabular}{|c|c|c|c|c|}
\hline Species & $\mathrm{FF}$ & DFT & diff. & RMSE \\
\hline $\mathrm{H} 2$ & 4623.4 & 4468.4 & 155.0 & 155.0 \\
\hline \multirow[t]{3}{*}{$\mathrm{CH} 2$} & 1818.2 & 1084.9 & 733.3 & 519.2 \\
\hline & 3600.0 & 3154.6 & 445.4 & \\
\hline & 3664.3 & 3394.7 & 269.5 & \\
\hline \multirow[t]{6}{*}{ CH3 } & 280.4 & 514.3 & -233.9 & 537.3 \\
\hline & 2192.0 & 1425.5 & 766.5 & \\
\hline & 2192.1 & 1425.5 & 766.6 & \\
\hline & 3562.0 & 3134.3 & 427.7 & \\
\hline & 3718.3 & 3318.7 & 399.6 & \\
\hline & 3718.4 & 3318.7 & 399.7 & \\
\hline \multirow[t]{9}{*}{$\mathrm{CH} 4$} & 1898.6 & 1355.9 & 542.7 & 465.4 \\
\hline & 1899.4 & 1355.9 & 543.5 & \\
\hline & 1902.1 & 1355.9 & 546.2 & \\
\hline & 2036.4 & 1569.6 & 466.8 & \\
\hline & 2037.4 & 1569.6 & 467.8 & \\
\hline & 3518.8 & 3049.7 & 469.1 & \\
\hline & 3526.8 & 3164.8 & 362.0 & \\
\hline & 3530.2 & 3164.8 & 365.4 & \\
\hline & 3539.0 & 3164.8 & 374.2 & \\
\hline \multirow[t]{3}{*}{$\mathrm{H} 2 \mathrm{O}$} & 2044.7 & 1617.1 & 427.6 & 261.1 \\
\hline & 3868.8 & 3848.8 & 20.0 & \\
\hline & 4105.2 & 3959.5 & 145.7 & \\
\hline $\mathrm{OH}$ & 4015.6 & 3763.0 & 252.6 & 252.6 \\
\hline \multirow[t]{6}{*}{$\mathrm{H} 2 \mathrm{CO}$} & 911.8 & 1207.4 & -295.7 & 937.0 \\
\hline & 1349.6 & 1269.1 & 80.5 & \\
\hline & 1937.4 & 1545.3 & 392.1 & \\
\hline & 3642.6 & 1785.7 & 1856.9 & \\
\hline & 3837.5 & 2930.3 & 907.1 & \\
\hline & 3859.2 & 2993.5 & 865.8 & \\
\hline \multirow[t]{4}{*}{$\mathrm{CHO}$} & 909.4 & - & 909.4 & 2326.9 \\
\hline & 1317.7 & 1115.1 & 202.5 & \\
\hline & 3552.6 & 1893.0 & 1659.6 & \\
\hline & 6369.4 & 2702.2 & 3667.1 & \\
\hline
\end{tabular}

Table S4: Vibrational frequencies of investigated species as calculated by ReaxF$\mathrm{F} /$ Agrawalla (FF) compared to B2PLYP-D3BJ (DFT) in $\mathrm{cm}^{-1}$ 


\begin{tabular}{|c|c|c|c|}
\hline Species & $\mathrm{FF}$ & DFT & diff. \\
\hline \multirow[t]{33}{*}{ Butanone } & 45.1 & 58.1 & -13.0 \\
\hline & 79.7 & 121.9 & -42.2 \\
\hline & 229.6 & 223.0 & 6.6 \\
\hline & 236.4 & 261.5 & -25.1 \\
\hline & 268.9 & 410.1 & -141.2 \\
\hline & 411.2 & 517.4 & -106.2 \\
\hline & 411.2 & 573.0 & -161.9 \\
\hline & 978.2 & 756.7 & 221.5 \\
\hline & 1131.0 & 793.5 & 337.5 \\
\hline & 1150.8 & 944.3 & 206.5 \\
\hline & 1213.6 & 970.2 & 243.5 \\
\hline & 1227.8 & 1013.1 & 214.7 \\
\hline & 1285.3 & 1106.3 & 178.9 \\
\hline & 1344.2 & 1131.7 & 212.6 \\
\hline & 1717.5 & 1240.5 & 476.9 \\
\hline & 1858.2 & 1298.3 & 559.9 \\
\hline & 1920.2 & 1351.3 & 568.9 \\
\hline & 1955.2 & 1398.8 & 556.4 \\
\hline & 1956.7 & 1425.9 & 530.8 \\
\hline & 1957.0 & 1479.9 & 477.2 \\
\hline & 2011.0 & 1482.2 & 528.7 \\
\hline & 2327.6 & 1497.1 & 830.5 \\
\hline & 2504.0 & 1519.2 & 984.7 \\
\hline & 2658.9 & 1520.7 & 1138.2 \\
\hline & 3418.0 & 1761.2 & 1656.8 \\
\hline & 3526.2 & 3044.9 & 481.3 \\
\hline & 3529.8 & 3055.0 & 474.9 \\
\hline & 3531.2 & 3056.8 & 474.4 \\
\hline & 3531.3 & 3115.7 & 415.6 \\
\hline & 3531.9 & 3121.4 & 410.5 \\
\hline & 3542.3 & 3129.8 & 412.5 \\
\hline & 3549.8 & 3137.9 & 411.9 \\
\hline & 4951.8 & 3168.1 & 1783.7 \\
\hline
\end{tabular}

Table S5: Vibrational frequencies of Butanone as calculated by ReaxFF/Agrawalla (FF) compared to B2PLYP-D3BJ (DFT) in $\mathrm{cm}^{-1}$. RMSE of FF to $\mathrm{QM}$ is $619.3 \mathrm{~cm}^{-1}$. 


\begin{tabular}{|c|c|c|c|c|}
\hline Reaction & $\mathrm{FF}$ & DFT & diff. & RMSE \\
\hline \multirow[t]{12}{*}{1} & $1807.9 \mathrm{i}$ & $1266.3 \mathrm{i}$ & $541.6 \mathrm{i}$ & 1661.3 \\
\hline & 70.5 & 537.2 & -466.7 & \\
\hline & 70.5 & 537.2 & -466.7 & \\
\hline & 1453.2 & 1078.4 & 374.8 & \\
\hline & 1453.2 & 1111.6 & 341.6 & \\
\hline & 1757.2 & 1111.6 & 645.6 & \\
\hline & 2014.8 & 1444.0 & 570.8 & \\
\hline & 2014.8 & 1444.0 & 570.8 & \\
\hline & 3592.2 & 1938.0 & 1654.2 & \\
\hline & 3639.7 & 3093.4 & 546.3 & \\
\hline & 3639.7 & 3243.6 & 396.1 & \\
\hline & 8523.2 & 3243.6 & 5279.6 & \\
\hline \multirow[t]{9}{*}{2} & $1999.8 \mathrm{i}$ & $987.2 \mathrm{i}$ & $1012.6 \mathrm{i}$ & 1950.7 \\
\hline & 31.5 & 408.3 & -376.8 & \\
\hline & 41.3 & 428.4 & -387.1 & \\
\hline & 492.0 & 809.4 & -317.4 & \\
\hline & 1488.4 & 1013.1 & 475.3 & \\
\hline & 2054.1 & 1208.1 & 846.0 & \\
\hline & 3633.0 & 2325.7 & 1307.4 & \\
\hline & 3708.8 & 3145.0 & 563.8 & \\
\hline & 8812.3 & 3347.9 & 5464.4 & \\
\hline \multirow[t]{6}{*}{3} & $1614.1 \mathrm{i}$ & $978.1 \mathrm{i}$ & $636.0 \mathrm{i}$ & 1265.4 \\
\hline & 477.3 & 516.1 & -38.8 & \\
\hline & 588.9 & 595.5 & -6.6 & \\
\hline & 1529.3 & 975.7 & 553.6 & \\
\hline & 4070.3 & 2845.4 & 1224.9 & \\
\hline & 6490.6 & 3771.2 & 2719.3 & \\
\hline \multirow[t]{4}{*}{4} & $1681.0 \mathrm{i}$ & $1564.8 \mathrm{i}$ & $116.2 \mathrm{i}$ & 3558.4 \\
\hline & 764.3 & 526.1 & 238.2 & \\
\hline & 773.6 & 839.7 & -66.0 & \\
\hline & 8925.4 & 1813.9 & 7111.6 & \\
\hline \multirow[t]{9}{*}{5} & $2104.9 \mathrm{i}$ & $1365.3 \mathrm{i}$ & $739.5 \mathrm{i}$ & 2415.8 \\
\hline & 77.2 & 340.1 & -262.8 & \\
\hline & 106.4 & 432.9 & -326.5 & \\
\hline & 665.1 & 1177.3 & -512.2 & \\
\hline & 916.7 & 1227.5 & -310.8 & \\
\hline & 1613.0 & 1332.8 & 280.2 & \\
\hline & 3325.2 & 1443.1 & 1882.1 & \\
\hline & 3838.5 & 1841.9 & 1996.6 & \\
\hline & 9522.6 & 2901.8 & 6620.9 & \\
\hline
\end{tabular}

Table S6: Vibrational frequencies of investigated transition states as calculated by ReaxFF/Agrawalla (FF) compared to B2PLYP-D3BJ (DFT) in $\mathrm{cm}^{-1}$ 


\begin{tabular}{|c|c|c|}
\hline$\overline{\mathrm{FF}}$ & DFT & diff. \\
\hline $2100.7 \mathrm{i}$ & -1950.1 & -150.6 \\
\hline 9.6 & 37.5 & -27.8 \\
\hline 24.0 & 51.5 & -27.5 \\
\hline 36.2 & 69.5 & -33.3 \\
\hline 134.7 & 105.7 & 29.0 \\
\hline 189.5 & 174.9 & 14.5 \\
\hline 228.3 & 212.4 & 15.9 \\
\hline 253.9 & 246.9 & 7.0 \\
\hline 308.0 & 384.5 & -76.5 \\
\hline 326.1 & 419.8 & -93.6 \\
\hline 409.2 & 502.5 & -93.3 \\
\hline 467.9 & 536.3 & -68.4 \\
\hline 794.6 & 602.3 & 192.3 \\
\hline 1000.0 & 643.9 & 356.1 \\
\hline 1170.1 & 778.9 & 391.3 \\
\hline 1213.5 & 785.5 & 428.0 \\
\hline 1276.7 & 943.7 & 333.0 \\
\hline 1298.5 & 963.7 & 334.8 \\
\hline 1317.2 & 975.2 & 342.0 \\
\hline 1392.1 & 1015.5 & 376.5 \\
\hline 1440.9 & 1104.2 & 336.7 \\
\hline 1630.0 & 1116.0 & 514.1 \\
\hline 1715.8 & 1139.1 & 576.7 \\
\hline 1776.0 & 1197.2 & 578.8 \\
\hline 1818.9 & 1293.2 & 525.7 \\
\hline 1843.1 & 1375.2 & 467.9 \\
\hline 1941.0 & 1392.9 & 548.1 \\
\hline 1956.0 & 1429.7 & 526.2 \\
\hline 1958.3 & 1443.5 & 514.8 \\
\hline 1983.2 & 1458.9 & 524.2 \\
\hline 2050.4 & 1471.8 & 578.6 \\
\hline 2486.0 & 1507.3 & 978.7 \\
\hline 2622.3 & 1514.2 & 1108.1 \\
\hline 2982.8 & 1745.9 & 1236.9 \\
\hline 3269.4 & 3036.9 & 232.5 \\
\hline 3521.3 & 3066.2 & 455.1 \\
\hline 3521.7 & 3073.2 & 448.5 \\
\hline 3522.7 & 3111.7 & 410.9 \\
\hline 3564.6 & 3138.7 & 425.8 \\
\hline 3576.6 & 3144.9 & 431.7 \\
\hline 3624.2 & 3216.0 & 408.1 \\
\hline 3641.3 & 3759.1 & -117.8 \\
\hline
\end{tabular}

Table S7: Vibrational frequencies of the TS of Butanone $+\mathrm{HO}_{2}$ as calculated by ReaxFF/Agrawalla (FF) compared to B2PLYP-D3BJ (DFT) in $\mathrm{cm}^{-1}$. RMSE of FF to QM is $460.9 \mathrm{~cm}^{-1}$. 


\section{Coordinates of Transition States}

The first column indicates the element and the second to fourth columns indicate the Cartesian coordinates.

\section{ReaxFF / Agrawalla}

Reaction 1: $\mathrm{CH}_{4}+\mathrm{H} \longleftrightarrow \mathrm{CH}_{3}+\mathrm{H}_{2}$

\begin{tabular}{rrrr}
\hline $\mathrm{C}$ & -0.000093 & -0.000092 & 0.280883 \\
$\mathrm{H}$ & 0.000333 & -1.065808 & 0.641089 \\
$\mathrm{H}$ & -0.923286 & 0.532234 & 0.641218 \\
$\mathrm{H}$ & 0.922469 & 0.533091 & 0.641570 \\
$\mathrm{H}$ & 0.000185 & 0.000186 & -1.177190 \\
$\mathrm{H}$ & 0.000391 & 0.000390 & -2.423667
\end{tabular}

Reaction 2: $\mathrm{CH}_{3}+\mathrm{H} \longleftrightarrow \mathrm{CH}_{2}+\mathrm{H}_{2}$

\begin{tabular}{rrrr}
\hline $\mathrm{C}$ & -0.478540 & 0.009978 & -0.129064 \\
$\mathrm{H}$ & -1.026571 & -0.939797 & 0.069341 \\
$\mathrm{H}$ & 1.016019 & -0.013091 & -0.065800 \\
$\mathrm{H}$ & -0.997228 & 0.975045 & 0.074367 \\
$\mathrm{H}$ & 2.239477 & -0.032151 & 0.052975
\end{tabular}

Reaction 3: $\mathrm{H}_{2} \mathrm{O}+\mathrm{H} \longleftrightarrow \mathrm{OH}+\mathrm{H}_{2}$

\begin{tabular}{rrrr}
\hline $\mathrm{H}$ & 0.235079 & 1.164902 & -0.097601 \\
$\mathrm{H}$ & 0.399175 & 2.194753 & -0.159931 \\
$\mathrm{H}$ & -0.294208 & -0.374845 & 0.888691 \\
$\mathrm{O}$ & 0.002812 & -0.271796 & -0.000102
\end{tabular}

Reaction 4: $\mathrm{OH}+\mathrm{H} \longleftrightarrow \mathrm{O}+\mathrm{H}_{2}$

$\begin{array}{rrrr}\mathrm{O} & -0.000000 & -0.000000 & 0.552139 \\ \mathrm{H} & -0.000012 & -0.000012 & -1.926287 \\ \mathrm{H} & -0.000007 & -0.000007 & -0.818288\end{array}$

Reaction 5: $\mathrm{H}_{2} \mathrm{CO}+\mathrm{H} \longleftrightarrow \mathrm{HCO}+\mathrm{H}_{2}$

\begin{tabular}{rrrr}
\hline $\mathrm{C}$ & -0.217029 & 0.309783 & -0.002049 \\
$\mathrm{H}$ & -1.645185 & -0.109394 & 0.007208 \\
$\mathrm{H}$ & -0.407891 & 1.409608 & 0.011604 \\
$\mathrm{O}$ & 0.840586 & -0.386281 & -0.019716 \\
$\mathrm{H}$ & -2.339887 & -1.128090 & 0.002959
\end{tabular}

Reaction $\mathrm{C}_{4} \mathrm{H}_{8} \mathrm{O}+\mathrm{HO}_{2} \longleftrightarrow \mathrm{C}_{4} \mathrm{H}_{7} \mathrm{O}+\mathrm{H}_{2} \mathrm{O}_{2}$

$\begin{array}{llll}\text { C } & 1.167022 & 3.664046 & -1.089927\end{array}$

C $\quad-0.085530 \quad 3.043192-0.385262$

C $\quad 0.226568 \quad 1.529209-0.082930$ 


$$
\begin{array}{llll}
\mathrm{C} & -1.043098 & 0.842862 & 0.381133 \\
\mathrm{H} & -0.853064 & -0.198714 & 0.749093 \\
\mathrm{H} & -2.031311 & 0.720259 & -0.739542 \\
\mathrm{H} & -1.588573 & 1.430034 & 1.165522 \\
\mathrm{H} & 1.003778 & 4.758629 & -1.329150 \\
\mathrm{H} & 1.391724 & 3.123844 & -2.059348 \\
\mathrm{H} & 2.082307 & 3.583122 & -0.428344 \\
\mathrm{H} & -1.000314 & 3.131470 & -1.035002 \\
\mathrm{H} & -0.315633 & 3.588029 & 0.572312 \\
\mathrm{O} & 1.401116 & 1.014753 & -0.272636 \\
\mathrm{O} & -2.982484 & 0.616781 & -1.830708 \\
\mathrm{O} & -3.495856 & 1.854518 & -2.024967 \\
\mathrm{H} & -4.299091 & 1.888665 & -1.425119
\end{array}
$$


B2PLYPD3-BJ/6-311 $++\mathrm{G}(\mathrm{d}, \mathrm{p})$

Reaction 1: $\mathrm{CH}_{4}+\mathrm{H} \longleftrightarrow \mathrm{CH}_{3}+\mathrm{H}_{2}$

\begin{tabular}{|rrrr|}
\hline $\mathrm{C}$ & 0.270658 & 0.000000 & 0.000018 \\
$\mathrm{H}$ & 0.515394 & -0.528671 & -0.915171 \\
$\mathrm{H}$ & 0.515271 & -0.528256 & 0.915480 \\
$\mathrm{H}$ & 0.515332 & 1.056927 & -0.000205 \\
$\mathrm{H}$ & -1.141723 & 0.000000 & -0.000077 \\
$\mathrm{H}$ & -2.028221 & 0.000000 & -0.000137 \\
\hline
\end{tabular}

Reaction 2: $\mathrm{CH}_{3}+\mathrm{H} \longleftrightarrow \mathrm{CH}_{2}+\mathrm{H}_{2}$

\begin{tabular}{|rrrr|}
\hline $\mathrm{C}$ & 0.000000 & 0.000000 & -0.285169 \\
$\mathrm{H}$ & 0.000000 & 0.972206 & -0.757158 \\
$\mathrm{H}$ & 0.000000 & 0.000000 & 1.191158 \\
$\mathrm{H}$ & 0.000000 & -0.972206 & -0.757158 \\
$\mathrm{H}$ & 0.000000 & 0.000000 & 2.034173 \\
\hline
\end{tabular}

Reaction 3: $\mathrm{H}_{2} \mathrm{O}+\mathrm{H} \longleftrightarrow \mathrm{OH}+\mathrm{H}_{2}$

\begin{tabular}{|rrrr|}
\hline $\mathrm{H}$ & -0.461292 & -0.976048 & 0.000000 \\
$\mathrm{H}$ & -0.491578 & -1.785233 & 0.000000 \\
$\mathrm{H}$ & 0.952869 & 0.141155 & 0.000000 \\
$\mathrm{O}$ & 0.000000 & 0.327516 & 0.000000 \\
\hline
\end{tabular}

Reaction 4: $\mathrm{OH}+\mathrm{H} \longleftrightarrow \mathrm{O}+\mathrm{H}_{2}$

\begin{tabular}{|lrrr|}
\hline $\mathrm{O}$ & 0.000000 & 0.000000 & -0.331917 \\
$\mathrm{H}$ & 0.000000 & 0.000000 & 1.773103 \\
$\mathrm{H}$ & 0.000000 & 0.000000 & 0.882236 \\
\hline
\end{tabular}

Reaction 5: $\mathrm{H}_{2} \mathrm{CO}+\mathrm{H} \longleftrightarrow \mathrm{HCO}+\mathrm{H}_{2}$

$\begin{array}{rrrr}\mathrm{C} & 0.124914 & 0.412393 & 0.000000 \\ \mathrm{H} & -0.935538 & 1.072670 & 0.000000 \\ \mathrm{H} & 1.041643 & 1.037687 & 0.000000 \\ \mathrm{O} & 0.124914 & -0.778860 & 0.000000 \\ \mathrm{H} & -1.854901 & 1.646168 & 0.000000\end{array}$

Reaction $\mathrm{C}_{4} \mathrm{H}_{8} \mathrm{O}+\mathrm{HO}_{2} \longleftrightarrow \mathrm{C}_{4} \mathrm{H}_{7} \mathrm{O}+\mathrm{H}_{2} \mathrm{O}_{2}$

$\begin{array}{rrrr}\mathrm{C} & -2.302463 & -1.284696 & -0.208871 \\ \mathrm{C} & -1.094616 & -0.711339 & 0.521401 \\ \mathrm{C} & -0.769947 & 0.717246 & 0.127740 \\ \mathrm{C} & 0.475748 & 1.299559 & 0.703260 \\ \mathrm{H} & 0.616483 & 2.359790 & 0.510482 \\ \mathrm{H} & 1.419034 & 0.706312 & -0.047997 \\ \mathrm{H} & 0.762504 & 0.961105 & 1.697863 \\ \mathrm{H} & -2.492537 & -2.309248 & 0.116129\end{array}$




\begin{tabular}{|rrrr}
$\mathrm{H}$ & -2.135908 & -1.289161 & -1.286992 \\
$\mathrm{H}$ & -3.194657 & -0.687481 & -0.017230 \\
$\mathrm{H}$ & -0.193391 & -1.309774 & 0.344878 \\
$\mathrm{H}$ & -1.246256 & -0.724020 & 1.607963 \\
$\mathrm{O}$ & -1.463971 & 1.370230 & -0.632312 \\
$\mathrm{O}$ & 2.268904 & 0.145022 & -0.635127 \\
$\mathrm{O}$ & 2.376588 & -1.117529 & -0.022816 \\
$\mathrm{H}$ & 3.160232 & -1.013923 & 0.535767
\end{tabular}

